\title{
Study on laser characteristics of Ho:YLF regenerative amplifiers: Operation regimes, gain dynamics, and highly stable operation points
}

\author{
P. Kroetz ${ }^{1,2,4} \oplus$ A. Ruehl ${ }^{3}$ A.-L. Calendron ${ }^{1,4,5} \cdot$ G. Chatterjee $^{1,2} \cdot$ H. Cankaya ${ }^{1,3,5}$. \\ K. Murari ${ }^{1,3,4}$ - F. X. Kärtner ${ }^{1,3,4,5}$ - I. Hartl ${ }^{1,3,5}$ • R. J. D. Miller ${ }^{1,2,5,6}$
}

Received: 23 December 2016 / Accepted: 20 March 2017 / Published online: 31 March 2017

(c) The Author(s) 2017. This article is an open access publication

\begin{abstract}
We present a comprehensive study of laser pulse amplification of Ho:YLF regenerative amplifiers (RAs) with respect to operation regimes, gain dynamics, and output pulse stability. The findings are expected to be more generic than for this specific gain material. Operation regimes are distinguished with respect to pulse energy and the appearance of pulse instability, and are studied as a function of the repetition rate, seed energy, and pump intensity. The corresponding gain dynamics are presented, identifying highly stable operation points related to highgain build-up during pumping and high-gain depletion during pulse amplification. Such operation points are studied numerically and experimentally as a function of several parameters, thereby achieving, for our Ho:YLF RA, highly stable output pulses with measured fluctuations of only $0.19 \%$ (standard deviation).
\end{abstract}

P. Kroetz

peter.kroetz@gmail.com

1 Center for Free-Electron Laser Science (CFEL), NotkestraSSe 85, 22607 Hamburg, Germany

2 Max-Planck Institute for the Structure and Dynamics of Matter (MPSD), Luruper Chaussee 149, 22761 Hamburg, Germany

3 Deutsches Elektronen-Synchrotron (DESY), Notkestrasse 85, 22607 Hamburg, Germany

4 Department of Physics, University of Hamburg, 22761 Hamburg, Germany

5 Centre for Ultrafast Imaging (CUI), Universität Hamburg, Luruper Chaussee 149, 22761 Hamburg, Germany

6 Departments of Chemistry and Physics, University of Toronto, Toronto M5S 1A7, Canada

\section{Introduction}

Pulse amplification with RAs is one of the most common approaches to amplify low energetic laser pulses to multi$\mathrm{mJ}$ pulse energies. Important for the design of any RA is its ability to generate energetically stable output pulses. Initially, observed instabilities in the output pulses from an RA were attributed to inherent noise originating from the pump sources [1]. However, later, it was reported that exceeding the repetition rate beyond a certain threshold value can also be a potential cause for large energy fluctuations $[2,3]$. This phenomenon, called bifurcation (or multifurcation), can cause output pulses to fluctuate between two (or more) values, and is an effect of the gain dynamics in RAs for consecutive pump and amplification cycles [3]. Figure 1 illustrates the output pulses of an RA that shows bifurcation as a function of the round trip (RT). The red dots represent a system without pump noise. Consequently, the pulse energies change strictly periodical between a high and a low value in the bifurcation, or are inherently stable at all other RTs. Once the RA is subject to pump noise, the pulse energies (green dots) are distributed over a larger range, notably not only in the bifurcation but also at the RTs at which the pulses were inherently stable without the pump noise. Although energetically stable output pulses are generally important for many applications, the basic understanding of the onset of bifurcation based on RA parameters such as pump intensity, seed fluence, and the number of RT, as well as the underlying gain dynamics remains incomplete. Similarly, even for RA operation at an inherently stable RT, there are no comprehensive studies and guidelines for an optimized noise performance, to the best of our knowledge.

The first experimental and numerical work dealing with the onset of bifurcation and chaotic output pulses was 


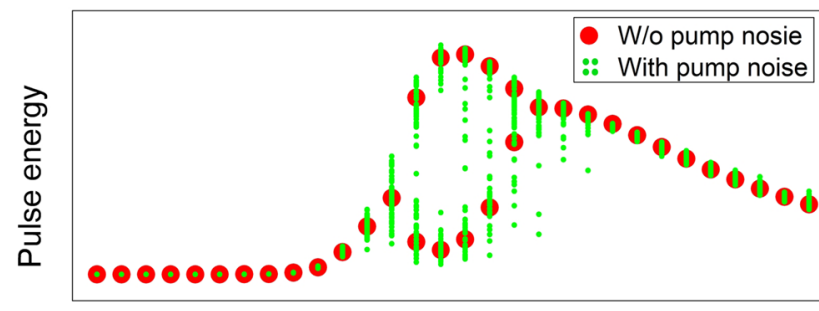

Round trip

Fig. 1 Illustration of the output pulses from an regenerative amplifier (RA) showing bifurcation, a periodical change of the output pulse energy between a high and low value, as a function of the (RT). Once pump noise is included, the output pulses are distributed over a larger range, even away from the bifurcation at RTs that previously produced stable output pulses

published by Dörring et al. [3] for a Yb:glass RA. The susceptibility of RAs to show unstable output pulses was connected to the repetition rate of the RAs and the excited state lifetime of the gain medium $\left(\tau_{\text {gain }}\right)$. For repetition rates on the order of the inverse lifetime $1 / \tau_{\text {gain }}$, RAs can show unstable output pulses.

It was later shown, but not quantified, that the seed energy also affects the onset of bifurcation, and that higher seed energies allow operation at higher repetition rates without the presence of bifurcation [4]. The first experimental hint that there is an inherently stable RT beyond the bifurcation instability, as illustrated in Fig. 1 at high RTs, was demonstrated in [3]. This was more precisely studied for an $\mathrm{Nd}: \mathrm{YVO}_{4} \mathrm{RA}$, showing bifurcation for a large range of RTs, prior to the start of further inherently stable RTs [5]. More recently, this was demonstrated, for the first time, for an Ho:YLF RA [6], achieving high pulse energies by overcoming bifurcation instability at a repetition rate of $1 \mathrm{kHz}$. Furthermore, the measurements and simulations showed that the output pulse stability is highest at a specific RT close to the maximum average output pulse energy, independent of the presence of bifurcation in the system at an earlier RT [6].

RAs lasing at a wavelength of $2 \mu \mathrm{m}$ and based, for example, on Ho:YLF, Ho:YAG [7], and Tm:YAP [8] have recently garnered attention as a viable driving source for mid-IR optical parametric amplifiers (OPAs) [9]. They can be used in conjunction with highly efficient non-oxide non-linear crystals (such as ZnGeP2 [9, 10]), as opposed to the more conventional $1-\mu \mathrm{m}$ pump sources. Unfortunately, Ho:YLF RAs, with an upper state lifetime of about $15 \mathrm{~ms}$ (or equivalently, an inverse lifetime of $\sim$ $67 \mathrm{~Hz}$ ), greatly suffer from the onset of bifurcation at the demonstrated repetition rates. Typically, Ho:YLF RAs were either operated at relatively low repetition rates [9], thus completely suppressing the onset of bifurcation, at high repetition rates until the onset of bifurcation [11], or directly in the bifurcation at a stable double-pulsing state [12]. In the latter case, pulse-picking only the higher energy pulse at half the repetition rate allows the extraction of stable and high-energy pulses $[12,13]$. By employing high pump intensities, we demonstrated operation of our Ho:YLF RA up to repetition rates of $750 \mathrm{~Hz}$ without any sign of bifurcation, which is more than an order of magnitude higher than the inverse lifetime [6]. This indicates that the pump intensity also plays a crucial role in the onset of bifurcation.

The numerical models that have been reported and used for the simulation of RAs either focused on a rather qualitative understanding of pulse instability and the underlying gain dynamics [3, 4], or on the reproduction of concrete laboratory measurements $[11,14,15]$. In the first case, rather simplistic mathematical expressions were used, neglecting for example an explicit dependence of the pump intensity on the gain build-up. In the second case, more complex and computationally intense numerical models were employed. Typically, they consist of a split-approach, simulating the pulse amplification with Frantz-NodvikFN equations and the gain build-up with rate-equations. RA operation was then considered as a function of few parameters in a rather limited parameter space. Hence, the reported findings provide, in both cases, only a partial understanding of the onset of pulse instability and the corresponding gain dynamics. As shown in Fig. 1, it is essential that any numerical model used to study pulse stability of RAs includes the effect of pump noise. Notably, there are no numerical studies for pulse amplification in RAs that include this noise source, to the best of our knowledge.

In this paper, we present a comprehensive, mostly numerical, study of pulse amplification with continuous wave $(\mathrm{CW})$-pumped Ho:YLF RAs in regards of operation regimes, gain dynamics, and output pulse stability. The simulation model is completely based on modified and computationally fast FN equations, allowing for an efficient variation of RA parameters in a large parameter space.

The simulations distinguish four different RA operation regimes exhibiting different output pulse characteristics as a function of the pump intensity, repetition rate, seed fluence, and pulse gain. These operation regimes represent inherent limitations in RA amplification in terms of stable output pulse energy and average output power. We analyze the onset of bifurcation and we empirically find that for high-gain RAs, the repetition rate at which bifurcation appears scales linearly with the pump intensity and follows a power law with the seed energy. We furthermore present the RA gain dynamics characterized by the gain build-up during the pump phase, and gain depletion during the pulse amplification. Upon adding pump noise to the simulations, we identify an operation point that offers the highest output pulse stability, in the following called high stability 
point (HSP), located at a high-gain build-up and high-gain depletion.

We analyze such HSPs numerically and experimentally as a function of various RA parameters. We thus achieve with our laboratory Ho:YLF RA highly stable output pulses with measured energy fluctuations of only $0.19 \%$ (standard deviation). Although this study was conducted with an Ho:YLF RA, the results are considered more generic and provide a more complete and general understanding of pulse amplification in RAs, independent of the gain material.

The paper has been structured in the following manner: Sect. 2 briefly describes the simulation model that is used for this study. In Sect. 3, the different RA operation regimes are distinguished and discussed, and the onset of bifurcation is studied as a function of the pump intensity and seed fluence. Section 4 describes the gain dynamics for the operation regimes and identifies HSPs. Analogous HSPs are studied numerically and experimentally as a function of various RA parameters in Sect. 5.

\section{Numerical simulation model}

This chapter summarizes the general simulation procedure used for the simulations in this paper. The model simulates consecutive pump and pulse amplification cycles, which allows a statistical analysis of a large ensemble of amplified output pulses. The model is sketched out in Fig. 2. At the beginning of the simulations, a random value for the initial inverted fraction $\beta$ in the laser crystal can be used as a starting value. During a pumping cycle, the inverted fraction

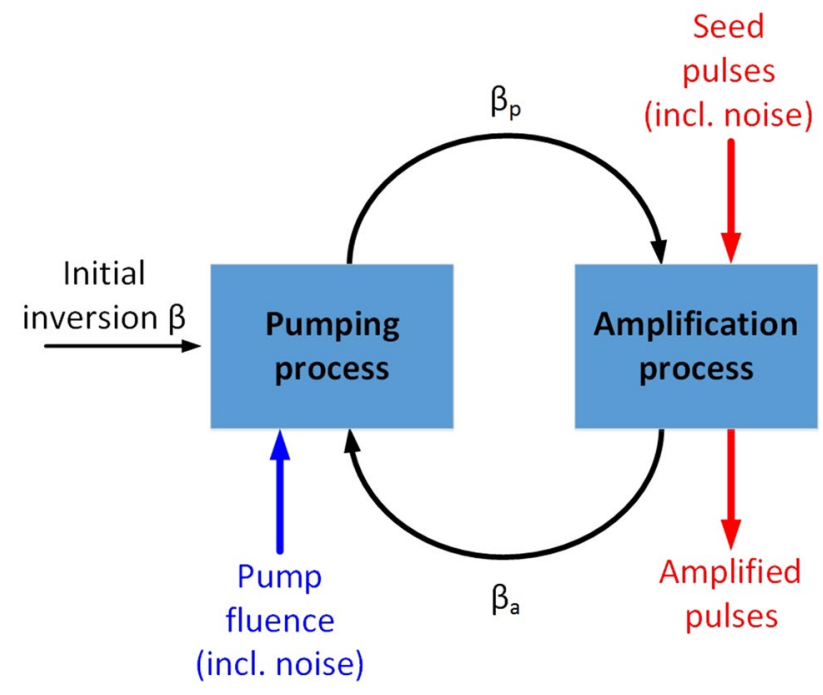

Fig. 2 Simulation of consecutive pump and amplification cycles. The inverted fraction $\beta_{\mathrm{p}}$ and $\beta_{\mathrm{p}}$ from the pump or amplification cycle is fed to the following amplification or pump cycle, respectively increases to the inverted fraction $\beta_{\mathrm{p}}$, which is then fed into the consecutive amplification cycle as the starting value. Then, during the amplification cycle, the inverted fraction decreases to the inverted fraction $\beta_{\mathrm{a}}$, which is again fed into the consecutive pumping cycle, and so on.

Noise originating from the pump and seed source is included by a Gaussian distributed variation of the pump and seed fluences for consecutive pump and amplification cycles. In this paper, we refer to the noise of the pump and seed source as the standard deviation of consecutive pump and seed fluences in percent of the corresponding mean fluence. Equivalently, the standard deviation of the output pulse fluence in percent of the mean output fluence is used as a measure for the output noise. The simulation framework was already presented in detail in [16], where the model was spectrally generalized and used to study spectral shaping effects in Ho:YLF RAs. Here, we used the monochromatic version as it is computationally less expensive. The equations used for this work can be found in Appendix 1 , along with further simulation details.

Although the simulation model is quite simplistic, it captures the essential physics necessary for this study. The model does not consider, for example, temporal or spatial effects, nor amplified spontaneous emission (ASE), upconversion, and thermal and non-linear effects. ASE could, for example, limit RA operation as it could cause a cavitydumped background when low seed pulse fluences and/or a high number of RTs are used.

However, despite these simplifications in the model, it was already successfully employed to reproduce the results from our Ho:YLF RA [6], including the onset and end of bifurcation instability, and the existence of a highly stable operation point close to gain depletion; the onset of bifurcation in dependence of the pump power [17]; and the operation of a burst-mode Yb:YAG laser [18]. The simulation parameters used for the simulations in this work are summarized in Appendix 3.

\subsection{Analysis of the gain dynamics}

To analyze the gain dynamics of RAs, the simulation results are analyzed in terms of the normalized gain $g_{\mathrm{p}}$ and the gain depletion $g_{\text {depl }}$. The normalized gain $g_{\mathrm{p}}$ represents the amount of stored energy in the gain medium after the pump process. It can have values between 0 and 1, where 0 represents no and 1 represents the maximum possible energy stored in the gain medium. The gain depletion $g_{\text {depl }}$ represents the amount of extracted energy during the pulse amplification. It can have values between 0 and 1 , where 0 represents no and 1 represents complete energy extraction. The equations used to calculate $g_{\mathrm{p}}$ and $g_{\text {depl }}$ can be found in the Appendix 3. 


\section{Operation regimes of regenerative amplifiers}

This chapter numerically studies pulse amplification in four characteristic operation regimes as a function of the repetition rate, pump intensity, seed pulse fluence, and pulse gain. The regimes were generally observed in experiments, for example, in $[5,19]$, but there has not been a study of these regimes as a function of the pump intensity, repetition rate, and seed fluence.

Figure $3 \mathrm{a}$ illustrates the four characteristic operation regimes (1)-(4) for regenerative amplification as a function of the repetition rate and pump intensity. The illustration is based on the corresponding simulations, which can be found in Appendix 4. The pump threshold (PT) indicates the pump intensity at which the amplifier starts providing gain to the seed pulses. Typical output pulse energies as a function of RT are sketched out for these regimes in Fig. 3b-e. The average output pulse energy is indicated by the solid red lines, and the optimum energy extraction points in these four regimes are highlighted with the yellow

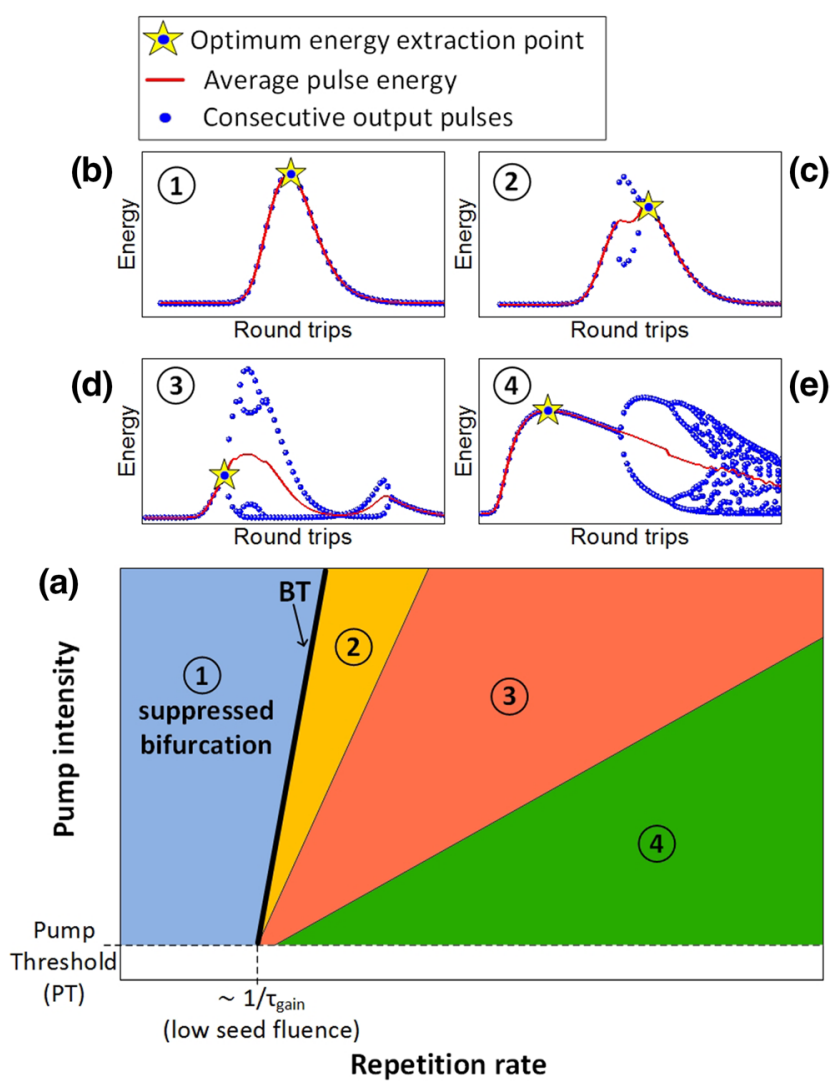

Fig. 3 a Illustration of RA operation regimes as a function of the repetition rate and pump intensity. b-e Typical output pulse energies for operation in these regimes, sketched out as a function of RT. The yellow starts indicate the optimum energy extraction points. The bifurcation threshold (BT) separating regimes (1) and (2) coincides with $\sim 1 / \tau_{\text {gain }}$ for low pump intensities and low seed energies. Illustration in $\mathbf{a}$ is based on the simulation results presented in Appendix 4 stars. These stars indicate maximum pulse energy extraction while avoiding bifurcation or pulse instability. Regime (1) allows a bifurcation-free operation, and stable pulses can be extracted at any RT. Although bifurcation appears for some RTs in regime (2), it is possible to extract high-energy output pulses at an inherently stable RT beyond the bifurcation instability, as indicated by the yellow star. In regime (3), the yellow star shifts to an RT before the pulse instability. Finally, in regime (4), the highest average output pulse energy can again be achieved stably. Our simulations predict that for later RTs, pulse instability can appear again, which was, however, not studied or described in [19] or in the literature, in general, to the best of our knowledge. It is important to note that for regimes (1), (2), and (4), the yellow stars coincide with the peaks of the solid red lines. For the following, we define the bifurcation threshold (BT) as the repetition rate that separates regime (1) and (2).

Figure 4a presents simulation results for BT as a function of the pump intensity and the seed fluence. The pump intensity is given in multiples of PTs. For three pump intensities $1 \times \mathrm{PT}, 5 \times \mathrm{PT}$, and $15 \times \mathrm{PT}$, we observe that for an increase in the seed fluence by 6 orders of magnitude (from 10 to $1000 \mathrm{~nJ} / \mathrm{cm}^{2}$ ), BT increases by a factor of $\sim 1.5$. For the complete range of seed fluences, BT increases by a factor of $\sim 4.5$ for an increase of the pump intensity from $1 \mathrm{xPTs}$ to $5 \mathrm{xPTs}$ and by a factor of $\sim 13$ for an increase from $1 \mathrm{xPTs}$ to $15 \mathrm{xPTs}$.

The results demonstrate that the simplistic assumption that $\mathrm{BT}$ is decided by the inverse lifetime of the gain medium $\left(1 / \tau_{\text {gain }}\right)$ is a limiting case for RAs with low pump intensity and low seed fluence. Based on the presented results, we propose the following proportionality between BT, the pump intensity, and the seed fluence

$\mathrm{BT} \propto \frac{1}{\tau_{\text {gain }}} \cdot I_{\text {pump }} \cdot J_{\text {seed }}^{b}$,

with $I_{\text {pump }}$ as the pump intensity, $J_{\text {seed }}$ as the seed pulse fluence and $\mathrm{b}$ as a fitting parameter. The relation suggests a linear dependence of BT with the pump intensity and a dependence on the seed fluence that follows a power law. To verify this assumption, we fitted the simulation results from Fig. 4a with

$\mathrm{BT}=\frac{1}{\tau_{\text {gain }}} \cdot f_{1}\left(I_{\text {pump }}\right) \cdot f_{2}\left(J_{\text {seed }}^{b}\right)$,

where the functions $f_{1}$ and $f_{2}$ represent the dependency on the pump intensity and the seed fluence with

$$
\begin{aligned}
& f_{1}=m \cdot\left(\frac{I_{\text {pump }}}{P T}-1\right)+n, \\
& f_{2}=c+d \cdot J_{\text {seed }}^{b},
\end{aligned}
$$




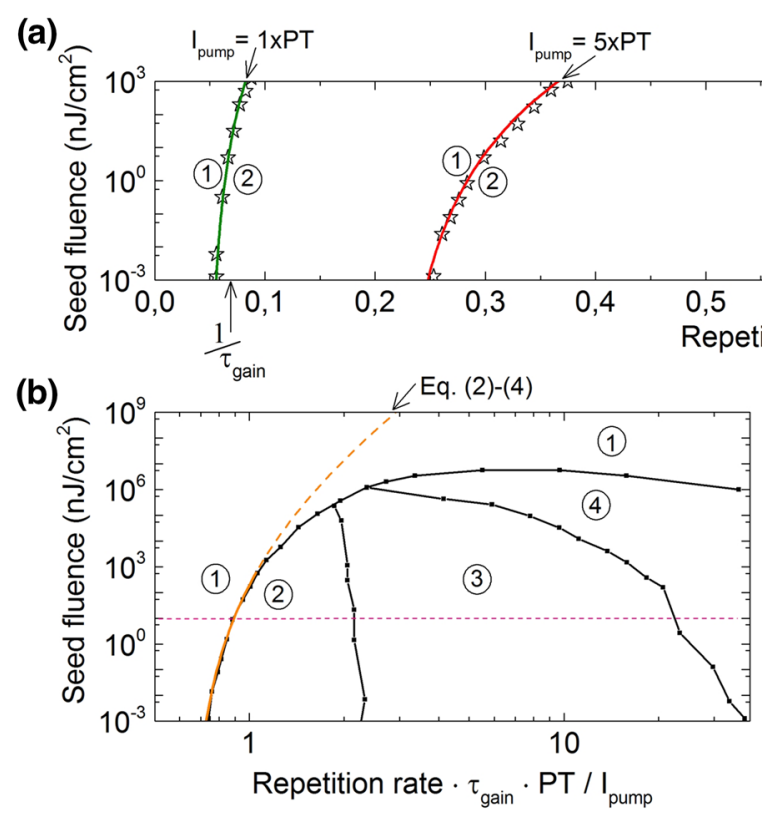

Fig. 4 a Simulation results for BT, separating regime (1) and (2), for the pump intensities $1 \times \mathrm{PT}, 5 \times \mathrm{PT}$, and $15 \times \mathrm{PT}$ and small seed fluences $(<<$ stored fluence in the gain medium). The solid lines in pink, red, and orange represent the fitted BT with Eqs. (2)-(4). The fitted parameter values are listed in Table 1 . b Operation regimes as a

Table 1 Parameter values for Eqs. (2)-(4), fitted to the simulation results for BT in Fig. $4 \mathrm{a}$

\begin{tabular}{ll}
\hline Parameter for $f_{1}$ & Parameter for $f_{2}$ \\
\hline$m=0.5$ & $c=1.29$ \\
$n=0.57$ & $d=4.53$ \\
& $b=0.12$ \\
\hline
\end{tabular}

with $m, n, c, d$, and $b$ as fitting parameters. The fitted BT curves are also shown in Fig. $4 \mathrm{a}$, with the solid lines. The fitted parameter values for $m, n, c, d$, and $b$ are listed in Table 1 . We find good agreement for the shown seed fluences that are much smaller than the total stored fluence in the gain medium, in this particular case, for seed fluences $<1000 \mathrm{~nJ} / \mathrm{cm}^{2}$. Figure $4 \mathrm{~b}$ presents the operation regimes (1)-4) for a pump intensity of $15 \times$ PT, now for a larger range of seed fluences and repetition rates. The repetition rate was normalized with the inverse excited state life time of the gain medium $1 / \tau_{\text {gain }}$ and the pump intensity (in multiples of PT). We observe that for seed fluences $>1000 \mathrm{~nJ} / \mathrm{cm}^{2}$, the simulated results for BT and the fitted curve (orange dashed line) start to deviate. For seed fluences exceeding a certain cut-off value (here $10^{6} \mathrm{~nJ} / \mathrm{cm}^{2}$ ), no pulse instability appears anymore (regime (2)), independent of the repetition rate.

Figure $4 \mathrm{c}$ presents the same data than Fig. $4 \mathrm{~b}$ but avoids the usage of the rather system specific seed fluence by evaluating the results in terms of the pulse gain. For many applications, operation at an intrinsically stable RT is preferred that coincide with the maximum extractable pulse energy (yellow stars coincide with the peak of the red lines in Fig. 3b-e), hence operation in regime (1), (2), or (4). The figure indicates that high gains exceeding $10^{6}$ can only be achieved in these regimes either for low normalized repetition rates of $\lesssim$ (for regime (1) and (2)) or for high normalized repetition rates of $\gtrsim 20$ (for regime (4)). We observe that there is a cut-off gain below which no pulse instability exists anymore (at any RT), observed for gains $\lesssim 100$. Similar to BT as a function of the seed fluence, we found that $\mathrm{BT}$ as a function of the gain also follows a power law.

\subsection{Typical output, normalized gain, and gain depletion values}

The horizontal pink dashed line in Fig. $4 \mathrm{~b}$ represented a seed fluence of $10 \mathrm{~nJ} / \mathrm{cm}^{2}$ (and a high-gain RA). Along this line, we consider in Fig. 5 the output at the optimum energy extraction points (illustrated by the yellow stars in Fig. 2). At these extraction points, the output pulse fluence and the average output pulse intensity (output pulse fluence $\mathrm{x}$ repetition rate) are plotted in Fig. $4 \mathrm{a}$ as a function of the normalized repetition rate. Figure $4 \mathrm{~b}$ shows the corresponding normalized gain $g_{\mathrm{p}}$ and the gain depletion $g_{\text {depl }}$, demonstrating that there are gain dynamics at play that are 

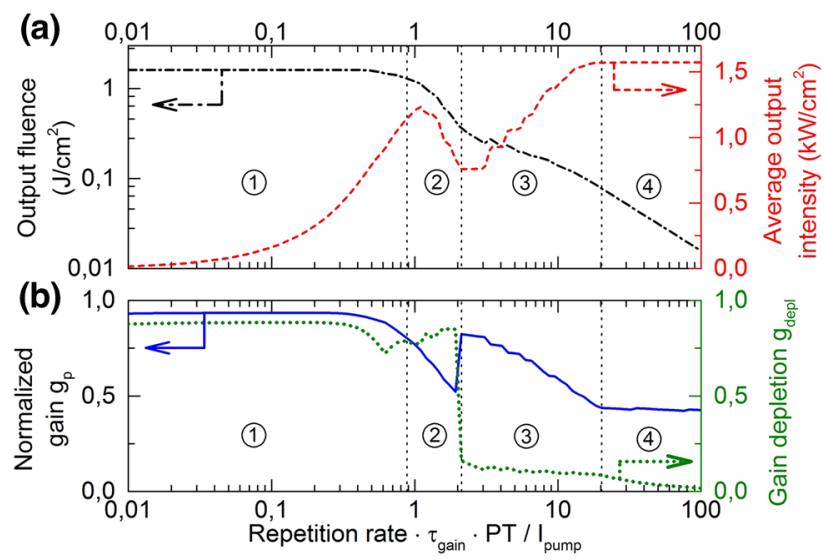

Fig. 5 a Output pulse fluence and the average output intensity, and b normalized gain and the gain depletion, as a function of the normalized repetition rate. The results represent operation of a high-gain $\mathrm{RA}$ along the pink dashed line in Fig. 4b, for operation at the corresponding optimum energy extraction points (indicated by yellow stars in Fig. 3b-e). The black dashed vertical lines represent the borders between the different operation regimes

characteristic for the regimes (1) to (4). The regimes are separated in the figure with the thin vertical dotted lines.

Regime (1) is characterized by a saturated normalized gain (blue solid line) and high-gain depletion (green dotted line), as shown in Fig. 4b. This means that a high fluence is stored in the gain medium during the pump process and most of it is depleted in the following amplification process. Consequently, high output fluences (black dashed-dotted line) can be extracted (Fig. 4a). Throughout regime (1), the output fluence is saturated and remains nearly constant with an increase in the repetition rate, while the average output intensity (red dashed line) increases almost linearly.

In regime (2), the pump intensity is no longer sufficient to saturate the normalized gain during the pump processes and, consequently, the output fluence decreases (black dashed-dotted line). Here, we observe the onset of bifurcation. The peak in the average output intensity (red dashed line) may represent the 'best' compromise between high output fluence extraction and high average output intensity. The transition between regimes (2) and (3) is marked by a sudden step in the normalized gain (blue solid line) and gain depletion (green dotted line). This corresponds to a jump of the yellow star from an RT after-pulse instability in Fig. 3c to an RT before-pulse instability in Fig. 3d. Consequently, less gain is depleted and the normalized gain after the successive pump processes increases.

The operation regimes (3) and (4) are characterized by low gain depletion and, consequently, low output fluence, as only a small amount of the stored fluence is depleted. We observe that the output fluence (black dashed-dotted line) decreases almost logarithmically with the repetition rate. At the beginning of regime (3), the average output intensity (red
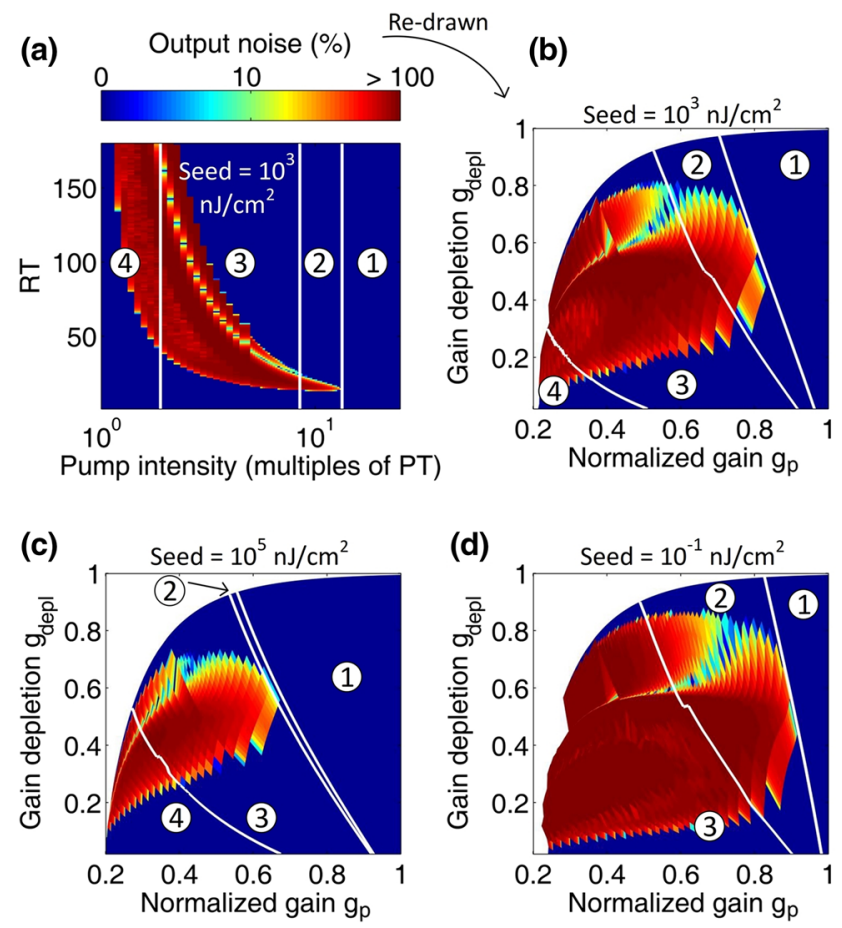

Fig. 6 a Output noise as a function of the pump intensity and RT, simulated with a seed fluence of $1000 \mathrm{~nJ} / \mathrm{cm}^{2}$. b Noise values from a evaluated and drawn as a function of the normalized gain and gain depletion. c Analog simulation results for the normalized gain and gain depletion simulated with a seed fluence of $10^{5} \mathrm{~nJ} / \mathrm{cm}^{2}$, and d with a seed fluence of $10^{-1} \mathrm{~nJ} / \mathrm{cm}^{2}$. The position of the operation regimes (1)-(4) shifts as a function of the seed fluence, as marked in the graphs

dashed line) is low but increases with an increase in the repetition rate. This is caused by the shift of the yellow star in Fig. 3d towards the peak of the average pulse energy (red line in Fig. 3e) as the repetition rate increases. In regime (4, the normalized gain (blue solid line) stays nearly constant, while the average output intensity (red dashed line) reaches its saturated maximum.

\section{Gain dynamics and high stability points HSPs}

The focus of this chapter lies on, first, exposing the actual gain dynamics during RA operation and, second, the identification of operation points with highly stable output pulse energies. The procedure to unravel the gain dynamics from the direct simulation results is explained in Figs. 6a and b. RA amplification was simulated as a function of the pump intensity and RT, and the corresponding output noise of consecutive output pulses was calculated, represented in Fig. $6 \mathrm{a}$ by the color code. There are areas that are inherently stable (in deep blue) 
and areas that show pulse instability (all other colors). The operation regimes (1)-(4) are marked accordingly in the figure.

For each value pair of RT and pump intensity (meaning for each pixel in Fig. 6a), the corresponding average normalized gain $g_{p}$ and gain depletion $g_{\text {depl }}$ was calculated (in accordance to the equations in Appendix 2) based on the inversion build-up and depletion recorded during the simulations. Figure $6 \mathrm{~b}$ presents the noise values from Fig. $6 \mathrm{a}$, now redrawn as a function of the corresponding gain values $g_{p}$, and $g_{\text {depl }}$, and hence, highlights the underlying gain dynamics. The spiky appearance of the border between the stable and the unstable area is an effect of the RT that can only be varied in the simulations in multiple integers of a single RT.

Figure $6 b-d$ demonstrates the effect of the seed fluence on the gain dynamics and the borders of the operation regimes. For an increase of the seed fluence from $1000 \mathrm{~nJ} /$ $\mathrm{cm}^{2}$ (in Fig. 6b) to $10^{5} \mathrm{~nJ} / \mathrm{cm}^{2}$ (in Fig. 6c), the inherently stable areas (in deep blue) grow. Contrary, for a decrease of the seed fluence to $10^{-1} \mathrm{~nJ} / \mathrm{cm}^{2}$ (in Fig. 6d), the inherently stable areas shrink. For the white areas in Fig. $6 \mathrm{~b}-\mathrm{d}$, the simulation did not produce values.

In general, the ranges producing inherently stable output pulses are characterized either by low gain depletion (little of the stored fluence extracted from the gain medium) or by high-gain depletion (most of the stored fluence extracted).

The simulations presented in Fig. 6 were conducted without pump noise and, hence, demonstrate the inherent susceptibility of an RA to show pulse instability. In the next step, the simulations of Fig. $6 \mathrm{a}$ and $\mathrm{b}$ were repeated, but now including noise from the pump source (simulated with 3\% standard deviation). The general RA gain dynamics are not affected by this rather small-scale pump intensity fluctuation, however, only by the inclusion of pump noise, the emergence of a specific highly stable operation point can be observed.

Figure $7 \mathrm{a}$ presents the results of the repeated simulations from Fig. 6a, including pump noise. The areas that were inherently stable in Fig. 6a (in deep blue) now also exhibit a certain amount of output noise. Notably, there is a specific area in Fig. 7a, labeled with high stability point (HSP), where the output noise is minimum (in deep blue). Figure $7 \mathrm{~b}$ demonstrates the corresponding gain dynamics. It demonstrates that the observed HSP is localized at a specific gain depletion value (in this case, $g_{\text {depl }} \sim 0.85$ ). The exact position mainly depends on the cavity losses and pump intensity, which will be discussed in Sect. 5. It is important to note, that the HSP can only be accessed if the pump intensities are sufficiently high to drive the RA into the operation regimes (1) or (2).
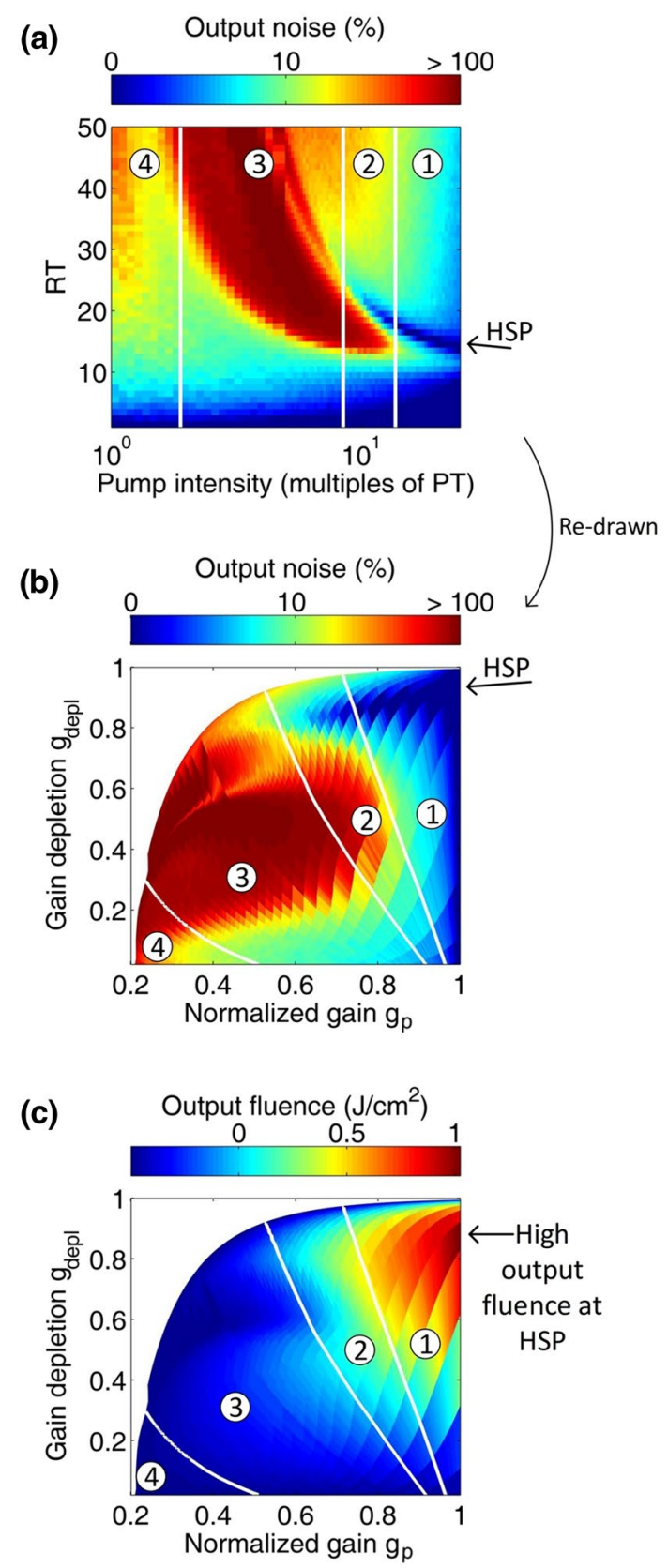

Fig. 7 a Output noise as a function of the pump intensity and the number of RT. The simulation includes pump noise, and all other parameters were chosen in accordance with the simulation in Fig. 6 a. b Noise values evaluated and drawn as a function of the normalized gain and gain depletion. For high normalized gain and high-gain depletion, the emergence of an HSP can be observed. c Corresponding output fluence as a function of the gain depletion and the normalized gain, indicating that the observed HSP is located at high output pulse fluences

Figure $7 \mathrm{c}$ demonstrates the corresponding output pulse fluence. It indicates that operation close to the HSP does 
not only yield the highest pulse stability, but also a high output pulse fluence.

\section{Noise optimization at high stability points (HSPs)}

The previous Sect. 4 demonstrated that the HSP corresponds to operation with high values of normalized gain and gain depletion (Fig. 7a, b). It seems plausible provided that sufficient pump intensity is employed to drive the system to high normalized gains $g_{\mathrm{p}}$, any parameter that allows for an adjustment of the gain depletion $g_{\text {depl }}$ should in principle allow to access the HSP.

This section presents both numerical and experimental results for an Ho:YLF RA that was optimized for operation at an HSP. In Sect. 5.1, the noise at the HSP is studied numerically as a function of the RA parameters RT, single-pass losses, pump intensity, and seed fluence. In Sect. 5.2, laboratory measurements for operation at an analogous HSP are presented as a function of the parameters RT, pump intensity, seed fluence, and crystal holder temperature.

As a measure for the output noise, we use as before the standard deviation of the output pulse fluence in percent of the mean output fluence (for the simulations), or an equivalent expression in terms of energy (for the measurements). The numerical and the experimental Ho:YLF laser were both operated in regime (1) however, they differ in their operational parameters. Consequently, the measurements focus on qualitative agreement with the simulations.

\subsection{Simulations}

The simulation approach is analogous to the approach in Sect. 4 to study the gain dynamics. RA operation was simulated as a function of various parameters and post-analysis of the simulation results yielded in the underlying gain dynamics.

We found that there is a different effect on the output noise, depending if the input noise is caused from the pump or the seed source. Figure 8 a shows the simulated output noise as a function of RT for three different combinations of pump and seed noise. Two ranges of operation can be distinguished:

(1) For up to RT $=13$, the gain is not yet depleted and the pulse energy increases with an increase in RT. Here, both the pump and the seed noise contributes to the output noise.

(2) Once the RA approaches the maximum pulse energy, the effect of the seed noise (green curve in Fig. 8a) becomes small compared to the effect of the pump
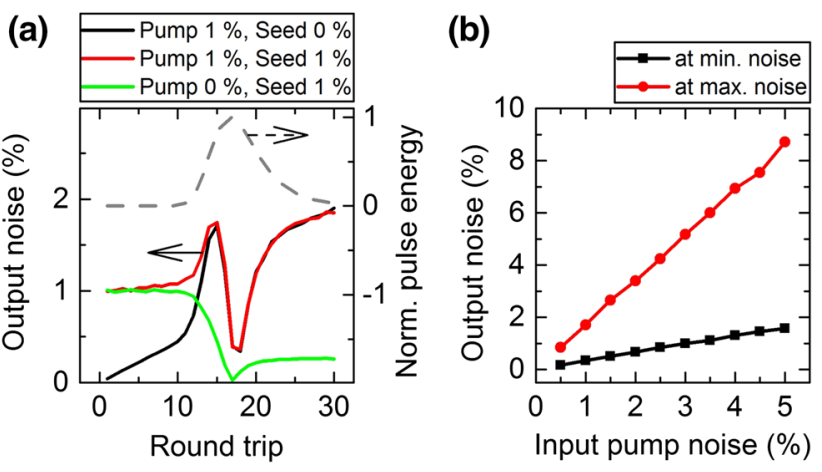

Fig. 8 a Output pulse noise and output pulse energy as a function of RT for three combinations of pump and seed noise. b Output pulse noise at $\mathrm{RT}=15$ (maximum noise) and $\mathrm{RT}=18$ (minimum noise) as a function of the input pump noise, demonstrating a linear influence

noise (black curve in Fig. 8a). In this range, the pump noise completely dominates the output noise, i.e., it becomes almost identical to that for the RA exhibiting both pump and seed noise (black and red line in Fig. 8a).

For the local maxima $(\mathrm{RT}=15)$ and minima $(\mathrm{RT}=18)$ of the output noise in Fig. 8a, the output noise was found to be linearly dependent on the input pump noise, as shown in Fig. 8b. Because of the observed linear dependence, all output noise values are normalized in the following with the input pump noise. The resulting 'normalized output noise' can be considered as the noise-suppression factor by which the input noise is suppressed in the output noise. A normalized output noise of 1 represents equal input pump and output noise. Figure $8 \mathrm{a}, \mathrm{b}$ emphasizes that to realize an RA that produces a highly stable output pulse energy, there is, besides operation at an HSP, also a need for a low-noise pump source. Furthermore, for the extraction of pulse energies close to gain depletion, a low-noise seed source is of less significance than a low-noise pump source.

In the following, we focus on the study of pulse amplification at HSPs. As seed noise can be neglected here, all simulations were conducted with pump noise only.

Figure 9a demonstrates the physical origin of HSPs. Figure 9a shows simulation results as a function of the pump intensity, for the three different single-pass losses 0,10 , and $15 \%$, for operation at a fixed RT (simulated at RT $=19$ ). Notably, the simulations were conducted as a function of the pump intensity and not as a function of RT. In terms of accessing an HSP, both parameters can be used, but only the pump intensity can be varied in sufficiently small steps to resolve the noise fine structure, as will be further emphasized in Fig. 10a and b. Figure 9a demonstrates that for a system without losses, no intermediate HSP is present. For increasing losses, the HSP 

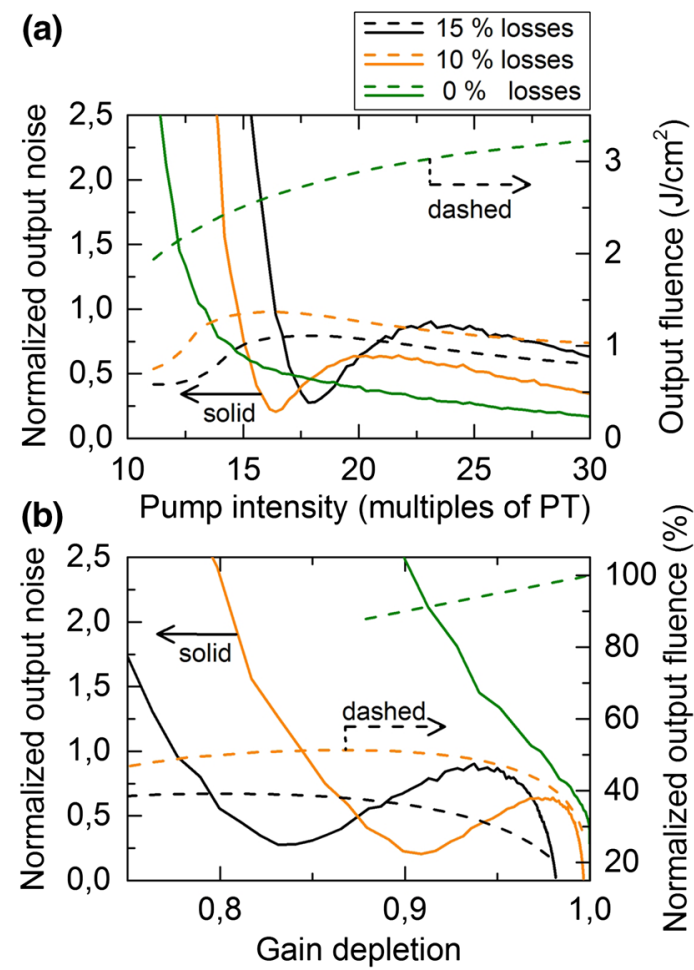

Fig. 9 a Normalized output noise as a function of the pump intensity for pulses coupled out at RT $=19$. b Same graph redrawn as a function of the gain depletion. The output fluence shown in a was additionally normalized in $\mathbf{b}$ to the stored fluence in the gain medium [Eq. (5)]. The simulations were conducted with different numbers of single-pass losses. Only the RAs with losses show an HSP (intermediate noise minimum)

becomes more and more pronounced. Consequently, the maximum output pulse fluence decreases with an increase in the losses.

By evaluation of the noise curves as a function of the gain depletion, as shown in Fig. 9b, one finds that the HSPs are located at specific gain depletion values, at higher depletions for lower losses. The output fluence in Fig. 9b was normalized to the total fluence that is stored in the gain material after the pump cycle with

$J_{\text {norm }}=\frac{J_{\text {out }}}{J_{\text {stor }}}$.

The interpretation is straightforward. For the loss-less system, the normalized output fluence increases linearly with the gain depletion and reaches a value of 1 for a gain depletion of 1 . In this case, the normalized output fluence is a direct representation of the gain depletion. On introducing losses, this direct representation is not valid anymore. In this specific case, simulating single-pass losses of 10 and $15 \%$ leads to a maximum normalized output fluence of 0.51 and 0.39 , respectively. This means that although most of the stored energy is extracted (represented by a high-gain
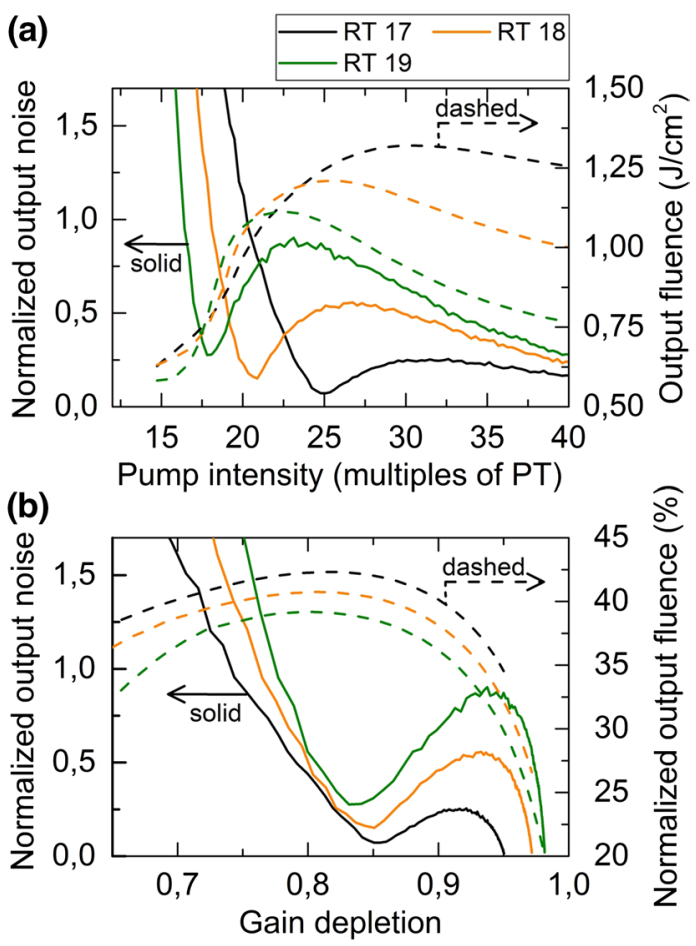

Fig. 10 a Normalized output noise and output fluence as a function of the pump intensity, for three different RT. b Re-plot of a as a function of the gain depletion. The output fluence shown in a was additionally normalized in $\mathbf{b}$ to the stored fluence in the gain medium [Eq. (5)]

depletion), a significant fraction of the stored energy (49 and $61 \%$ ) is lost and dissipated into the environment.

Figures 10 and 11 further investigate the accessibility and characteristics of HSPs. Figure 10a shows the normalized output noise and output fluence as a function of the pump intensity, for three different RTs. It demonstrates that for a decrease in the RT, the HSP shifts to higher pump intensities. Most importantly, we observe that for an increase in the pump intensity, the RA can produce output pulses with a higher stability (lower output noise at the corresponding HSPs).

These curves are redrawn in Fig. 10b as a function of the gain depletion, with the output fluence normalized according to Eq. (5). When comparing Fig. 10a and b, we find that for higher pump intensities, the HSPs are located at higher gain depletions and provide an increased output fluence and normalized output fluence. We assume that the main reasons for the increase in the output fluences are twofold. They are, first, due to an increase in the stored fluence in the gain medium (due to the higher pump intensity) and, second, due to a decrease in the accumulated losses (due to the lower RT).

In the following, we try to quantify these contributions by comparing to output fluence in Fig. 10a and the 

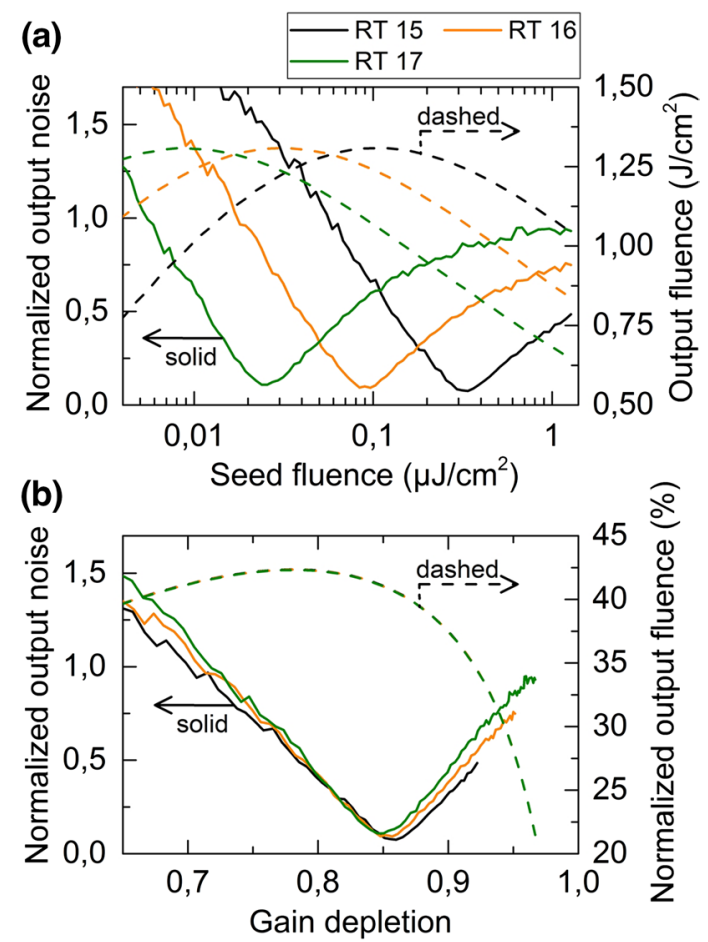

Fig. 11 a Normalized output noise and output fluence as a function of the seed fluence, for three different RTs. b Re-plot of $\mathbf{a}$ as a function of the gain depletion. The output fluence shown in a was additionally normalized in $\mathbf{b}$ to the stored fluence in the gain medium [Eq. (5)]

normalized output fluence in Fig. 10b. While the output fluence is affected by the pump intensity and the accumulated losses, the normalized output fluence is mainly affected by the accumulated losses alone (due to its normalization to the stored fluence in the gain medium). For operation at an HSP, we observe that the maximum normalized output fluence increases in Fig. 10b by $\sim 8 \%$ (comparing RT $=17$ with $\mathrm{RT}=19$ ). In comparison, the output fluence in Fig. 10a increases by $\sim 18 \%$, slightly more than twice as much. Hence, we conclude for this specific RA that the $8 \%$ increase is contributed by the decreased accumulated losses and the $10 \%$ (the difference between the 18 and the $8 \%$ ) is contributed by the increased stored fluence.

Analogous to Fig. 10, Fig. 11 shows results for RA operation as a function of the seed fluence for three RTs. Figure 11a presents the normalized output noise and output fluence, and Fig. 11b evaluates and presents the same data as a function of the gain depletion. Analog to the results in Fig. 10, increasing RT shifts the HSP to a decreased seed pulse fluence.

Rather counter-intuitively, when operating at RT $=17$ instead of RT $=15$, the output fluence and the normalized output fluence both increase by approximately $1.5 \%$ despite more than an order of magnitude less seed fluence (at the corresponding HSP). This is rather unexpected, as one would assume that an increase in RT leads to a decrease in the output pulse fluence due to an increase in accumulated losses. However, we believe that this observation is valid as long as the seed fluence is small compared to the total stored fluence in the gain medium and other loss channels (such as ASE or spontaneous inversion decay during pulse amplification) are negligible. For high-gain RAs with longlifetime gain materials, such as for Ho:YLF, this precondition is fulfilled in most cases. The above observation can be made traceable as follows:

(1) Considering a loss-less system, the stored fluence in the gain medium represents the maximum output pulse fluence that can be extracted. Unlike higher pump intensities, higher seed fluences do not change the stored fluence in the gain medium.

(2) When losses are included, the extractable output fluence is lowered by the accumulated losses. The simulations suggest, however, that a lower or higher seed fluence does not change the absolute amount of losses. This can be seen in Fig. 11d, where the lines for the normalized output fluence for the different RTs overlap each other.

(3) From Fig. 11d, it can be seen for higher RTs that the output fluence at the corresponding HSP shifts closer to the maximum of the output fluence curve. This leads to an increase in the output fluence for lower seed fluences if the RA is operated at the corresponding HSP. This behavior agrees with measurements as well, which are discussed in Fig. 12c.

\subsection{Measurements}

We operated our Ho:YLF RA [6] in regime (1) (at a repetition rate of $100 \mathrm{~Hz}$ ) and measured the output noise and the output pulse energy as a function of various parameters. The RA was seeded with pulses from an Ho:fiber oscillator [20] that produced pulses centered around $2053 \mathrm{~nm}$ with a bandwidth of $7 \mathrm{~nm}$. The pulses were stretched with a chirped volume Bragg grating prior seeding. The RA was pumped with a commercial CW Tm:fiber laser that produces up to $120 \mathrm{~W}$ of randomly polarized light. Unfortunately, the output power of this laser could not be tuned in fine steps. The measurements presented in Fig. 12a and b, however, required a fine tuning of the pump power. This was realized by a polarization of the pump laser output and by usage of a further polarizer and $\lambda / 2$-waveplate. The measurements that did not require a variation of the pump power, in Fig. 12c and d, were conducted with $18 \mathrm{~W}$ of the unpolarized pump laser output. To avoid water condensation at low crystal temperatures, the complete RA setup was purged with a constant flow of dry air, keeping the 

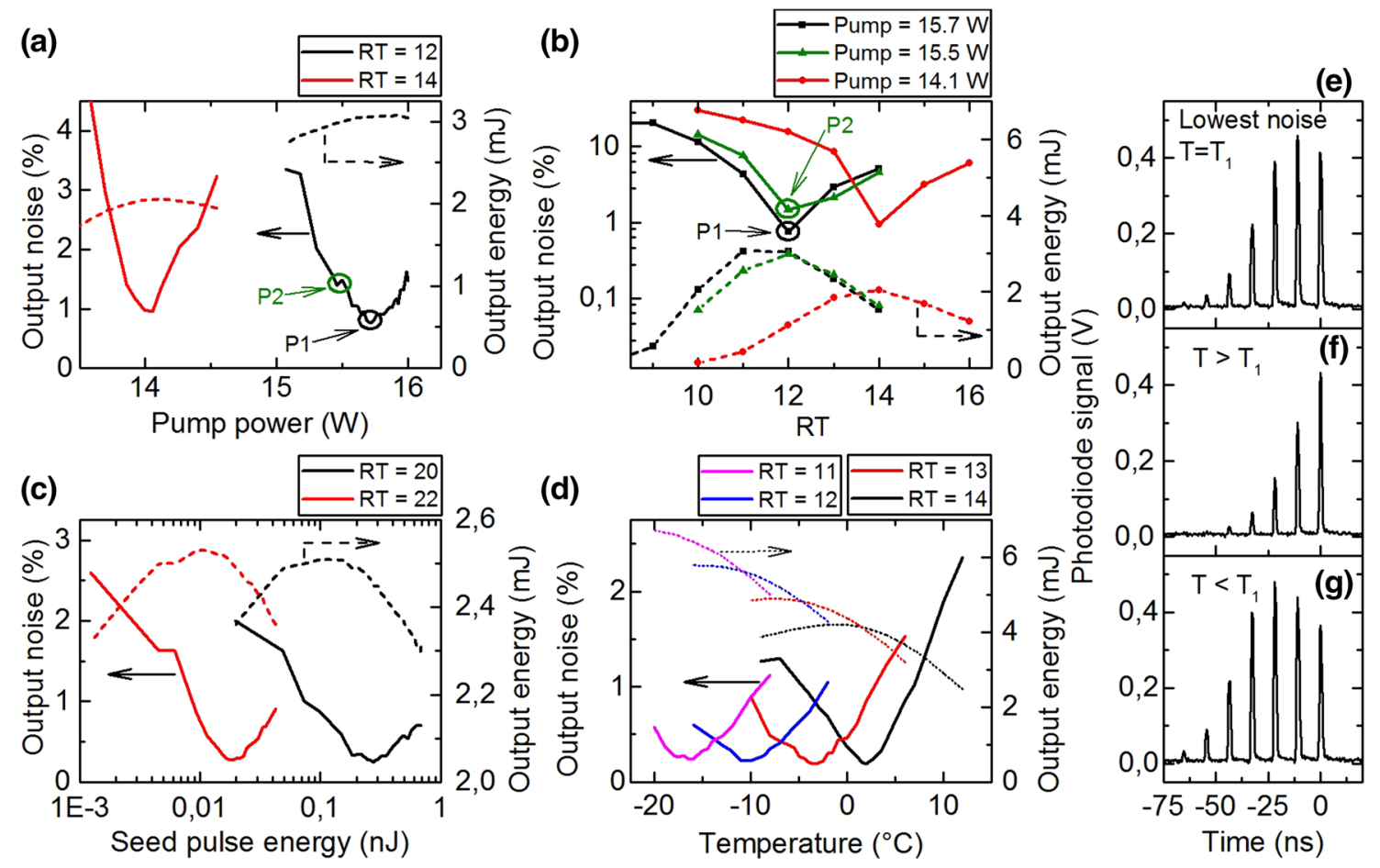

Fig. 12 Measured output noise and pulse energy as a function of a pump power, b RT, c seed pulse energy, and d crystal holder temperature. e, f intra-cavity pulse built-up for operation at, before and after the HSP, respectively, controlled by the crystal holder temperature

humidity level $<2 \%$. We observed that purging the RA cavity also significantly improved the stability of the amplified output pulses, related to an improved pump laser stability under dry atmosphere (see Appendix 5, Fig. 14e). If not specified otherwise, the RA was seeded with pulse energies of $690 \mathrm{pJ}$ and the temperature of the thermo-electrically controlled Ho:YLF crystal holder was kept at $18{ }^{\circ} \mathrm{C}$. Further details about the experimental Ho:YLF RA setup can be found in Appendix 5.

The presented pulse energies correspond to the uncompressed output, measured with a commercial calibrated energy meter. For each measurement, 3000 consecutive output pulses were recorded ( $30 \mathrm{~s}$ measuring time at a repetition rate of $100 \mathrm{~Hz}$ ) and analyzed with respect to pulse energy and output noise. Figure 12a presents the measured output noise and output pulse energy as a function of the pump power for $\mathrm{RT}=12$ and $\mathrm{RT}=14$. The minimum noise values are 0.76 and $0.95 \%$ for 12 and 14 RTs, respectively. As predicted by the simulations in Fig. 10a, for an increase in the pump power, the amplified output pulses can be coupled out at an earlier RT. At the corresponding HSPs, the output noise decreases and the output pulse energy increases for an increase in the pump power. In this particular case, the stability was improved by $\sim 20 \%$ and the pulse energy increased by $\sim 50 \%$. The range of pump powers for operation at an HSP can be quite narrow. This is demonstrated in Fig. 12a with the two points P1 and P2, representing, for $\mathrm{RT}=12$, the output noise for the pump powers $15.7 \mathrm{~W}$ and $15.5 \mathrm{~W}$, respectively. On a reduction of the pump power by only $1.3 \%$ (from P1 with $15.7 \mathrm{~W}$ to P2 with $15.5 \mathrm{~W}$ ), the output noise increased by $\sim 100 \%$, from 0.76 to $1.49 \%$.

Figure $12 \mathrm{~b}$ demonstrates the output noise and output energy as a function of RT, for the pump powers $15.7 \mathrm{~W}$, 15.1 W and 14.1 W. The two points P1 and P2 represent the same points than in Fig. 12a. The figure emphasizes that, while for operation at $15.7 \mathrm{~W}$, the lowest noise HSP can be accessed for $\mathrm{RT}=12$, for a pump power of $15.5 \mathrm{~W}$, there is no RT with a similar low output noise. The reason is that due to the function principle of RAs, the RT can only operated with multiple integers of a single RT, and hence, cannot be fine-tuned. Consequently, the lowest HSP cannot be accessed anymore if the pump power is slightly off its optimum value and RT is the only other parameter used to control the RA.

Figure 12c studies the effect of the seed pulse energy on the output noise and the output energy for different RTs. As suggested by the simulations in Fig. 11a, we observe a slightly lower noise at the HSP for an increase in the seed energy, at a lower RT. Furthermore, the output energy at the corresponding HSPs shifts closer to the maximum of the corresponding output pulse energy curve. This leads to the counter-intuitive effect that at the corresponding HSPs, the output pulse energy increases 
despite lower seed energy. In particular, for operation at the HSPs, we measured noise values of 0.24 and $0.27 \%$ for the seed pulse energies of 280 and $19 \mathrm{pJ}$, with corresponding output pulse energies of 2.45 and $2.5 \mathrm{~mJ}$, respectively. In this case, operation at the higher RT resulted in a stability improvement of $11 \%$. At the same time, despite more than an order of magnitude more seed energy, the amplified output energy decreased by $2 \%$.

During operation with the Ho:YLF RA, we experimentally discovered that a variation of the Ho:YLF crystal holder temperature allows a noise optimization similar to the optimization described in dependence of the pump power and seed energy. Figure $12 \mathrm{~d}$ shows the output noise and the pulse energy as a function of the crystal holder temperature for RT $=11$ to $\mathrm{RT}=14$. Analog to the previous noise curves in Fig. 10a-c, there is a distinct noise minimum, in this case at a specific crystal holder temperature. For a decrease in the crystal holder temperature, we also observe a significant increase in the output pulse energy. With a decrease in the crystal holder temperature from 18 to $-16^{\circ} \mathrm{C}$, the output pulse energy increases almost linearly by a factor of 2.6 , from 2.5 to $6.43 \mathrm{~mJ}$.

Currently, the temperature dependence of the emission and absorption cross sections is not implemented in the simulation model. However, based on the observations and findings described in this paper, we propose the following physical interpretation. We observe that with a decrease in the Ho:YLF crystal temperature, the seed pulses experience a stronger amplification, caused by higher absorption cross sections at the pump wavelength and higher emission cross sections at the seed wavelength at lower temperatures [9, 21]. Consequently, the HSPs (optimum gain depletion) is reached with fewer RTs and the pulses can be coupled out earlier. Hence, the output energy increases at lower temperatures due to fewer accumulated losses.

We observed that the minimum noise values measured with linearly polarized light were higher by up to a factor of 3 than the ones measured with randomly polarized light. These noise values, however, cannot be directly compared as the actual noise from the pump laser might be different for the two cases. Unfortunately, no noise values for the unpolarized and polarized pump laser output were available, but we expect two circumstances to contribute to the increased RA output noise observed:

(1) The pump laser was operated for Fig. 12a and $12 \mathrm{~b}$ at $32 \mathrm{~W}$, almost twice the value used for the measurements with random polarization at $18 \mathrm{~W}$ in Fig. 12c and $d$. Therefore, the higher noise level of the RA output could be explained with different noise levels of the pump.
(2) The fraction of the polarized light that is used to pump the RA could fluctuate, thus introducing an additional source of pump noise.

Figure 10e-g shows recorded oscilloscope traces for the intra-cavity pulse build-up typical for operation at a noise minimum and for operation before and after the minimum (with a less or more depleted gain), respectively. These operation points were accessed by controlling the crystal holder temperature while keeping RT fixed. We observe that for $\mathrm{T}=\mathrm{T} 1$ (in Fig. 12e), the output pulses exhibit the lowest noise, whereas the pulses exhibit higher noise for $\mathrm{T}<\mathrm{T} 1$ and $\mathrm{T}>\mathrm{T} 1$ (in Fig. 12f, g).

\section{Conclusions}

We presented a comprehensive analysis of pulse amplification in Ho:YLF RAs. Although this study was conducted with an Ho:YLF RA, the findings are expected to be more generic and lead to a more complete understanding of RA operation regimes, gain dynamics, and low-noise operation.

The employed numerical simulation model, based on the iterative Frantz-Nodvik(FN) formalism, simulates consecutive pump and amplification cycles. The model also accounts for noise originating from the pump and seed sources. The model is computationally fast, which is important for simulations involving a large number of consecutive pump and amplification cycles. This is crucial for a statistical analysis of results when RA parameters are varied finely or over a large range.

In Sect. 3, we numerically identified RA operation regimes with respect to the repetition rate, pump intensity, seed fluence, and pulse gain. We recognized four different operation regimes that show different output pulse characteristics. In regime (1), for low repetition rates, no bifurcation instability exists. In regime (2), bifurcation appears, but the highest average pulse energy is at an inherently stable RT beyond the bifurcation instability. In regime (3), the highest output energy at an inherently stable RT is limited due to the onset of pulse instability. In regime (4), the highest average pulse energy can be extracted at an RT beforepulse instability appears, and consequently, the instability can be practically ignored under such circumstances. Our simulations showed that the bifurcation threshold (BT), separating regimes (1) and (2), shifts to higher repetition rates for increased pump intensities and higher seed fluences. The results indicate that the simplistic assumption that the onset of bifurcation is decided by the inverse lifetime of the gain medium $\left(1 / \tau_{\text {gain }}\right)$ is a limiting case for an RA with low pump intensity and high-gain (low seed fluence). We empirically find for high-gain RA with gains $>10^{6}$, a linear 
proportionality between $\mathrm{BT}$ and the pump intensity, and a proportionality following a power law for the seed fluence.

In Sect. 4, we presented a numerical analysis of the gain dynamics in RAs operating in regimes (1)-(4) with respect to gain build-up and gain depletion in the gain medium. On including noise in the simulations, we observed the emergence of HSPs that allow the extraction of highly stable output pulses with a high pulse energy. This operation point corresponds to a high-gain build-up during RA pump and a high-gain depletion during pulse amplification.

In Sect. 5, we numerically and experimentally studied the output noise and output pulse energy at HSPs. The fundamental cause for the observed HSPs was numerically identified to be the RA losses. This means for every real laboratory RA that is operated in the regimes (1) or (2) that there always is an HSPs located in close proximity to the highest output pulse energy. Our findings demonstrate that any RA parameter that allows a fine-adjustment of the gain depletion can be used to access and optimize these HSPs. To experimentally study analogous HSPs with an Ho:YLF RA, we varied the pump intensity, seed fluence, number of round trips, and crystal holder temperature. Optimization of these parameters led to highly stable output pulses from our Ho:YLF RA with measured pulse energy fluctuations of only $0.19 \%$ (standard deviation). The noise curves show very characteristic noise minima that can be quite narrow (noise increases by $100 \%$ for a change in the pump power of only $\sim 1.3 \%$ ). Therefore, the lowest noise operation points can be easily missed if the RA operational parameters are chosen as slightly different from their optimum values, or if they drift with time and cause the RA output noise to move up and down the corresponding noise curves.

Acknowledgements Open access funding provided by Max Planck Society. The authors thank Haider Zia for helpful discussions.

Open Access This article is distributed under the terms of the Creative Commons Attribution 4.0 International License (http:// creativecommons.org/licenses/by/4.0/), which permits unrestricted use, distribution, and reproduction in any medium, provided you give appropriate credit to the original author(s) and the source, provide a link to the Creative Commons license, and indicate if changes were made.

\section{Appendix 1: The FN equations and the simulation model}

The simulation model used in this work utilizes the classical monochromatic Frantz-Nodvik (FN) formalism [15, 22, 23], which is a common approach to simulate pulse amplification in a laser gain media, as long as the amplification process is short compared to the upper state lifetime of the excited ions. Typically, this means that $\mathrm{CW}$ pumping cannot be considered with this set of equations. In this work, the equations were modified to include the inversion decay losses by considering the inversion decay law [24], allowing for the simulation of $\mathrm{CW}$ pumped laser gain media with an excited state life time of $\gtrsim 7 \mathrm{~ms}$ [16].

The complete simulation model was comprehensively presented in [16], but in contrast to [16] where the equations were further modified to the spectral regime, for this work here, the classical monochromatic FN equations were used. In the following, the general simulation approach and the used equations are summarized. Equations (6)-(10) describe a single-pass of an incoming fluence through the gain medium. The fluence can be either amplified or absorbed (depending on the emission and absorption cross sections $\sigma_{\mathrm{em}}$ and $\sigma_{\mathrm{em}}$ at the considered wavelength) and the inverted fraction decreases or increases from $\beta_{i-1}$ to $\beta_{i}$, respectively, as calculated by Eqs. (11)-(14). The correction term to account for the inversion decay [Eq. (14)] typically can be neglected for amplification and pump processes that are much shorter than the excited state lifetime.

The single-pass gain of a quasi-three level gain medium can be calculated with

$G_{i-1}=\exp \left(g_{i-1} l\right)$,

with the small signal gain

$g_{i-1}=\sigma_{\text {eff }, i-1} N$,

and the effective gain cross section

$\sigma_{\text {eff }, i-1}=\beta_{i-1}\left(\sigma_{\mathrm{em}}+\sigma_{\mathrm{abs}}\right)-\sigma_{\mathrm{abs}}$,

where $\beta_{i-1}$ represents the inverted fraction, $\sigma_{\mathrm{em}}$ and $\sigma_{\mathrm{abs}}$ the emission and absorption cross sections at the considered wavelengths, $N$ is the dopant ion density in the gain media, and $l$ is the length of the gain medium.

After a single passage of an incoming fluence $\mathrm{J}_{i-1}$, the fluence after the passage $\mathrm{J}_{i}$ can be calculated with

$\left.J_{i}=J_{\text {sat }} T \ln \left(1+G_{i-1} \exp \left(\frac{J_{i-1}}{J_{\text {sat }}}\right)-1\right)\right)$,

with the saturation fluence

$J_{\mathrm{sat}}=\frac{h c}{\lambda\left(\sigma_{\mathrm{abs}}+\sigma_{\mathrm{em}}\right)}$,

and $T$ as the single-pass transmission to generally account for losses in the system. To update the previous gain $g_{i-1}$ to the new average gain $g_{i}$, the following relation is used:

$g_{i}=\frac{J_{\text {stor }, i-1}-\left(\frac{J_{i}}{T}-J_{i-1}\right)}{J_{\text {sat }} l}=g_{i-1}-\frac{\frac{J_{i}}{T}-J_{i-1}}{J_{\text {sat }} l}$,

with the stored fluence

$J_{\text {stor }, i-1}=J_{\text {sat }} \ln \left(G_{i-1}\right)=\sigma_{\text {eff }, i-1} N l J_{\text {sat }}$. 
Rearranging Eq. (8) and Eq. (12), the new inverted fraction $\beta_{i}$ can be calculated by

$\beta_{i}=\frac{\frac{J_{s t o r, i}}{J_{s a t} l N}+\sigma_{a b s}}{\sigma_{\mathrm{em}}+\sigma_{a b s}}$.

To account for the inversion decay losses during the pump processes, the incoming pump fluence is temporally divided by slicing it into smaller fluence slices with a time duration of $\Delta t$ each. Each slice passes through the gain medium separately, and the inverted fraction is consecutively updated. After each fluence slice, the inverted fraction is corrected by the inversion decay law [24]

$\beta_{i}^{*}=\beta_{i} \exp \left(-\frac{\Delta t}{\tau_{\text {gain }}}\right)$.

\section{Appendix 2: The normalized gain $g_{p}$ and gain depletion $g_{\text {depl }}$}

The gain dynamics in this work were analyzed in terms of the normalized gain $g_{\mathrm{p}}$ and the gain depletion $g_{\text {depl }}$, which represent the gain build-up during the pump phase and the gain extraction during pulse amplification, respectively. In the following, on the right-hand side of Eqs. (15)-(17), we use the superscripted index ${ }^{a}$ and ${ }^{p}$ to indicate post-amplification and post-pumping values, respectively. We defined the normalized gain $g_{\mathrm{p}}$ as

$$
\begin{aligned}
g_{\mathrm{p}} & =\frac{J_{\text {stor }}^{p}\left(\lambda_{\mathrm{a}}\right)}{J_{\text {stor,pTrans }}\left(\lambda_{\mathrm{a}}\right)}, \\
& =\frac{\sigma^{p}{ }_{\text {eff }}\left(\lambda_{\mathrm{a}}\right)}{\sigma_{\text {eff }, p \text { Trans }}\left(\lambda_{\mathrm{a}}\right)}, \\
& =\frac{\beta^{p}\left(\sigma_{\mathrm{em}}\left(\lambda_{\mathrm{a}}\right)+\sigma_{\mathrm{abs}}\left(\lambda_{\mathrm{a}}\right)\right)-\sigma_{\mathrm{abs}}\left(\lambda_{\mathrm{a}}\right)}{\left(\beta_{\mathrm{pTrans}}\right)\left(\sigma_{\mathrm{em}}\left(\lambda_{\mathrm{a}}\right)+\sigma_{\mathrm{abs}}\left(\lambda_{\mathrm{a}}\right)\right)-\sigma_{\mathrm{abs}}\left(\lambda_{\mathrm{a}}\right)},
\end{aligned}
$$

with

$\beta_{\text {pTrans }}=\frac{\sigma_{a b s}\left(\lambda_{\mathrm{p}}\right)}{\sigma_{\mathrm{em}}\left(\lambda_{\mathrm{p}}\right)+\sigma_{a b s}\left(\lambda_{\mathrm{p}}\right)} \exp \left(-\Delta t / \tau_{\text {gain }}\right)$.

where $\sigma_{g}^{p}\left(\lambda_{\mathrm{a}}\right)$ represents the effective gain cross section at the amplification wavelength $\lambda_{\mathrm{a}}$, and $\beta^{p}$ is the inverted fraction after the pump process. $\sigma_{\mathrm{g}, \mathrm{p} \text { Trans }}\left(\lambda_{\mathrm{a}}\right)$ represents the highest possible effective gain cross section at the amplification wavelength. In the frame of our FN based simulation model, this happens for an inverted fraction $\beta_{\mathrm{pTrans}}$ at which the gain medium becomes transparent for the pump wavelength. For slicing the pump fluence into a high number of time slices (meaning small $\Delta \mathrm{t}$ ), the term $\exp \left(-\Delta t / \tau_{\text {gain }}\right)$ can be neglected. A normalized gain of $g_{\mathrm{p}}=1$ represents the maximum possible inverted fraction in the gain medium that can be achieved during a pump process, and it equally represents the maximum possible stored fluence.

As a measure for the extracted fraction of that stored fluence after the amplification process, we define the gain depletion $g_{\text {depl }}$ as

$$
\begin{aligned}
g_{\text {depl }} & =1-\frac{J^{a}{ }_{\text {stor }}\left(\lambda_{\mathrm{a}}\right)}{J^{p} \text { stor }}\left(\lambda_{\mathrm{a}}\right) \\
& =1-\frac{g^{a}\left(\lambda_{\mathrm{a}}\right)}{g^{p}\left(\lambda_{\mathrm{a}}\right)}=1-\frac{\sigma_{\text {eff }}^{a}\left(\lambda_{\mathrm{a}}\right)}{\sigma^{p}{ }_{\text {eff }}\left(\lambda_{\mathrm{a}}\right)} \\
& =1-\frac{\beta^{a}\left(\sigma_{\mathrm{em}}\left(\lambda_{\mathrm{a}}\right)+\sigma_{\mathrm{abs}}\left(\lambda_{\mathrm{a}}\right)\right)-\sigma_{\mathrm{abs}}\left(\lambda_{\mathrm{a}}\right)}{\beta^{p}\left(\sigma_{\mathrm{em}}\left(\lambda_{\mathrm{a}}\right)+\sigma_{\mathrm{abs}}\left(\lambda_{\mathrm{a}}\right)\right)-\sigma_{\mathrm{abs}}\left(\lambda_{\mathrm{a}}\right)} .
\end{aligned}
$$

The gain depletion lies between 0 and 1 , where 0 represents no energy extraction during pulse amplification and 1 represents a complete extraction of all stored energy. In the case of an RA without losses, the output fluence is proportional to $g_{\mathrm{p}}$ and $g_{\text {depl }}$ :

$J_{\text {out }} \propto g_{\mathrm{p}} \cdot g_{\text {depl }}$.

\section{Appendix 3: Simulation parameter}

For all simulations in this work, we used the same basic RA parameters, except specified otherwise. We used a beam diameter for the pump and cavity modes in the gain crystal of $1 \mathrm{~mm}$. The gain crystal was simulated with a length of 1 $=10 \mathrm{~mm}$ and the Holmium doping concentration was $1 \%$ (Holmium dopant ion density $N=1.2 \times 10^{20} \mathrm{~cm}^{-3}[25]$ ). The seed fluence was $10 \mathrm{~nJ} / \mathrm{cm}^{2}$ and the pump was simulated as linearly polarized, by utilizing the literature values for the absorption and emission cross sections at the pump and seed wavelengths for $\pi$-polarization [26]. We always took a value of $15 \%$ for single-pass losses (represented by $T=0.85$ ), except for the simulations to study the effect of the losses in Fig. 9. The pump threshold $(1 \times \mathrm{PT})$ was found to be $4.5 \mathrm{~W}$. All simulations were conducted for a repetition rate of $1 \mathrm{kHz}$, except for the simulations as a function of it. For all simulations that included pump noise, a pump noise value of 3\% (standard deviation of the consecutive pump fluences) was used. An overview of the used simulation parameters can be found in Table 2 .

To decouple the simulation results from the starting conditions, we neglected a certain number of pump and amplification cycles in the analysis. This number depends on the type of analysis conducted. The simulations to distinguish RA operation regimes were performed without pump and seed noise and show the inherent susceptibility of the RA to unstable output pulses. Once the initial inversions were chosen appropriately for the starting conditions, we observe that simulating 10 pump and amplification cycles and neglecting the first 5 cycles generally allow the distinction of the operation regimes. In contrast, to statistically study 
Table 2 Simulation parameters

\begin{tabular}{lllll}
\hline Figure & Pump power in PT & Repetition rate $(\mathrm{kHz})$ & Seed fl. $\left(\mathrm{nJ} / \mathrm{cm}^{2}\right)$ & Pump noise $(\%)$ \\
\hline 4a & $1 / 5 / 15$ & $0.03-3$ & $10^{-3}-10^{3}$ & 0 \\
4b & 15 & $0.03-30$ & $10^{-3}-10^{9}$ & 0 \\
4c & 15 & $0.03-30$ & $10^{-3}-10^{9}$ & 0 \\
$5 \mathrm{a}$ & 15 & $0.01-100$ & 10 & 0 \\
$5 \mathrm{~b}$ & 15 & $0.03-100$ & 10 & 0 \\
$6 \mathrm{a}$ & $0-55$ & 1 & $10^{3}$ & 0 \\
$6 \mathrm{~b}$ & $0-55$ & 1 & $10^{3}$ & 0 \\
$6 \mathrm{c}$ & $0-55$ & 1 & $10^{5}$ & 0 \\
$6 \mathrm{~d}$ & $0-55$ & 1 & $10^{-1}$ & 0 \\
$7 \mathrm{a}$ & $0-55$ & 1 & $10^{3}$ & 3 \\
$7 \mathrm{~b}$ & $0-55$ & 1 & $10^{3}$ & 3 \\
$7 \mathrm{c}$ & $0-55$ & 1 & $10^{3}$ & 3 \\
$8 \mathrm{a}$ & 22 & 1 & 10 & $0-1$ \\
$8 \mathrm{~b}$ & 22 & 1 & 10 & $0-5$ \\
$9 \mathrm{a}$ & $11-67$ & 1 & 10 & 3 \\
$9 \mathrm{~b}$ & $11-67$ & 1 & 10 & 3 \\
$10 \mathrm{a}$ & $11-67$ & 1 & 10 & 3 \\
$10 \mathrm{~b}$ & $11-67$ & 1 & 10 & 3 \\
$11 \mathrm{a}$ & 22 & 1 & 10 & 0 \\
$11 \mathrm{~b}$ & 22 & $0.03-100$ & $1-10^{3}$ & 3 \\
13 & $0-55$ & & 10 & 3 \\
\hline
\end{tabular}

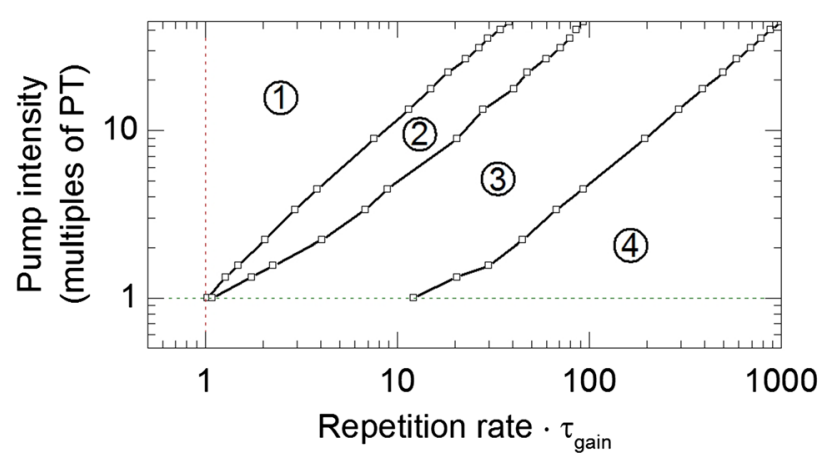

Fig. 13 Operation regimes as a function of the pump intensity and the repetition rate

the noise of the output pulses when pump noise is included, a larger number of pump and amplification cycles were simulated. Here, we always simulated 1500 cycles and neglect the first 100 cycles.

\section{Appendix 4: Operation regimes as a function of the pump power and repetition rate}

Figure 13 presents the simulation results based on which Fig. 3 (illustration of the operation regimes as a function of the pump power and the repetition rate) was drawn. For better visibility of the borders and the regimes, the axes for the pump intensity and repetition rate were logarithmically scaled.

\section{Appendix 5: The Ho:YLF RA setup}

Figure 14a-d presents details of the Ho:YLF RA setup that was used for the measurements in Sect. 5.2. The oscillator pulses [20] to seed the RA were stretched with a CVBG in a double-pass configuration (not shown in the figure). After stretching, the pulse energy was $690 \mathrm{pJ}$ with a spectral bandwidth of $3.25 \mathrm{~nm}$. Based on the specified chirp rate of $41 \mathrm{~nm} / \mathrm{ps}$ for the CVBG, we estimated the pulses to be chirped to a pulse duration of $267 \mathrm{ps}$.

Due to the recent commercial availability of high power and large aperture Faraday rotators for the wavelength of $2 \mu \mathrm{m}$ (FastPulse Technology, Inc.), the RA cavity is arranged linearly (shown in Fig. 14a). The pump chain, shown in Fig. 14c, consists of two telescopes and an optional polarization splitting attenuator. The simulated path of rays is shown in Fig. 14b. The telescopes image the end of the pump fiber into a $4 \mathrm{~cm}$-long Ho:YLF crystal that has a Holmium doping concentration of $1 \%$. The image diameter in the Ho:YLF crystal is $1 \mathrm{~mm}$ and can 
(a) Cavity mode

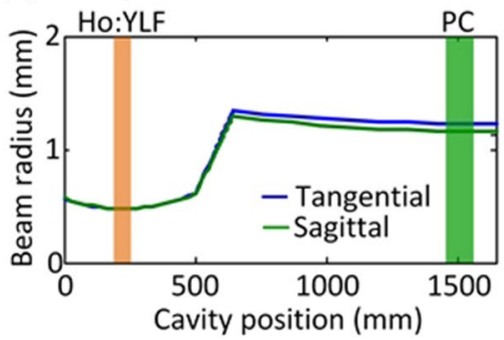

(b) Pump chain imaging

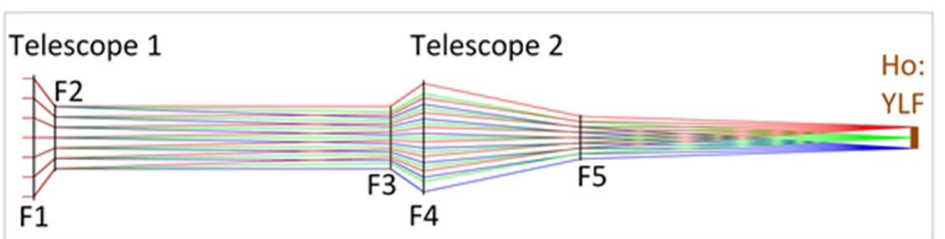

(c) Pump chain

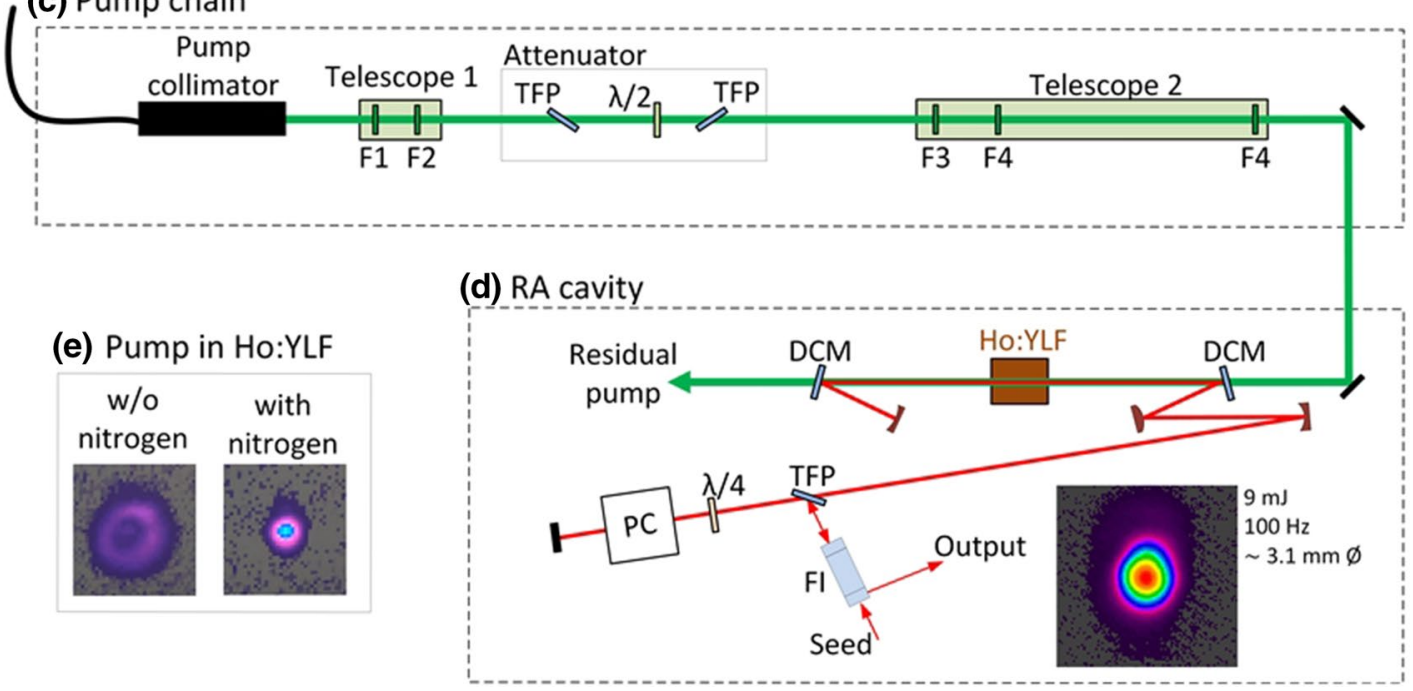

Fig. 14 Schematic of the Ho:YLF RA setup. a Simulated cavity mode. $\mathbf{b}$ Simulated path of rays of the pump laser, creating an image of the end of the pump fiber in the Ho:YLF crystal. c Pump chain consists of a polarization splitting attenuator (optional) and two telescopes that adjust the image size of the pump fiber in the Ho:YLF

be adjusted by simply moving the lenses F3 and F4 of Telescope 2 .

A typical output beam shape, with a beam diameter of $\sim 3.1 \mathrm{~mm}$, is also shown in Fig. 14d. It was measured in a distance of about $2.5 \mathrm{~m}$ after the RA output port and after being compressed with a CVBG (not shown in the sketch). In this specific case, the uncompressed output pulse energy was $9 \mathrm{~mJ}$ at a repetition rate of $100 \mathrm{~Hz}$. A randomly polarized pump power of $\sim 24 \mathrm{~W}$ was used. The simulated mode in the Ho:YLF crystal has a radius of $475 \mu \mathrm{m}$, resulting in a peak pulse fluence in the crystal of $\sim 2.5 \mathrm{~J} / \mathrm{cm}^{2}$. We did not observe crystal damage for these parameters.

During RA operation, the setup was constantly purged with dry air to avoid condensation of water on the Ho:YLF crystal and because the output pulse stability of the RA was observed to be significantly improved under dry atmosphere. This improved stability can be connected to a significantly more stable and improved pump beam. A typical pump spot in the Ho:YLF crystal plane under laboratory and under dry atmosphere is shown in Fig. 14e. The disturbed pump beam shape might be attributed to the water crystal. c Breadboard setup of the RA cavity. e Typical pump beam spot in the Ho:YLF crystal plane for the RA setup under laboratory atmosphere and for the setup purged with dry air. $D C M$ dichroic mirror, TFP thin film polarizer, $\lambda / 4$-wave plate, $P C$ pockels cell, FI Faraday isolator

vapor in the laboratory atmosphere that has absorption lines close to the lasing wavelength of the pump laser at $1940 \mathrm{~nm}$.

In this paper, measured results were presented for the RA operated in operation regime (1). Results for this setup operated in regimes (1) and (2) were published in [6]. Operation in regime (4) could not be demonstrated with the current setup, as a cavity-dumped background appeared for high repetition rates, which necessitates a high number of RTs. Higher seed pulse energies can potentially overcome this limitation.

\section{References}

1. J.E. Swain, F. Rainer, IEEE J. Quantum Electron 5, 385 (1969)

2. X.D. Wang, P. Basséras, J. Sweetser, I.A. Walmsley, R.J.D. Miller, Opt. Lett. 15, 839 (1990)

3. J. Dörring, A. Killi, U. Morgner, A. Lang, M. Lederer, D. Kopf, Opt. Express 12, 1759 (2004)

4. M. Grishin, V. Gulbinas, A. Michailovas, Opt. Express 15, 9434 (2007) 
5. M. Grishin, V. Gulbinas, A. Michailovas, Opt. Express 17, 15700 (2009)

6. P. Kroetz, A. Ruehl, G. Chatterjee, A.-L. Calendron, K. Murari, H. Cankaya, P. Li, F.X. Kärtner, I. Hartl, R.J.D. Miller, Opt. Lett. 40, 5427 (2015)

7. P. Malevich, T. Kanai, H. Hoogland, R. Holzwarth, A. Baltuska, A. Pugzlys: CLEO: 2015, OSA Technical Digest (online) (Optical Society of America, 2015), paper SM1P.4 (2015)

8. A. Wienke, D. Wandt, U. Morgner, J. Neumann, D. Kracht, Opt. Express 23, 16884 (2015)

9. M. Hemmer, D. Sánchez, M. Jelínek, V. Smirnov, H. Jelinkova, V. Kubeček, J. Biegert, Opt. Lett. 40, 451 (2015)

10. P. Malevich, G. Andriukaitis, T. Flöry, A. Verhoef, A. Fernández, S. Ališauskas, A. Pugžlys, A. Baltuška, L. Tan, C. Chua, P. Phua, Opt. Lett. 38, 2746 (2013)

11. L. von Grafenstein, M. Bock, U. Griebner, T. Elsaesser, Opt. Express 23, 14744 (2015)

12. L. von Grafenstein, M. Bock, G. Steinmeyer, U. Griebner, T. Elsaesser, Laser Photon. Rev. 10, 123 (2016)

13. T. Metzger, A. Schwarz, C.Y. Teisset, D. Sutter, A. Killi, R. Kienberger, F. Krausz, Opt. Lett. 34, 2123 (2009)

14. P. Raybaut, F. Balembois, F. Druon, P. Georges, IEEE J. Quantum Electron. 41, 415 (2005)

15. H.J. Teunissen, Multipass amplifier for Terawatt Ti:sapphire laser system. Ph.D. thesis, University Twente (2007)
16. P. Kroetz, A. Ruehl, K. Murari, H. Cankaya, F.X. Kärtner, I. Hartl, R.J.D. Miller, Opt. Express 24, 9905 (2016)

17. P. Kroetz, A. Ruehl, G. Chatterjee, K. Murari, H. Cankaya, A.-L. Calendron, F. X. Kaertner, R.J.D. Miller: CLEO: 2015, OSA Technical Digest (online) (Optical Society of America, 2015), paper SF1F.3 (2015)

18. F. Moglia, P. Kroetz, S. Koehler, L. Winkelmann, I. Hartl, In 7th EPS-QEOD Europhoton Conference 2016, paper SS-2.7-Wed-p9 (2016)

19. P. Gao, H. Lin, J. Li, J. Guo, H. Yu, H. Zhang, X. Liang, Opt. Express 24, 13963 (2016)

20. P. Li, A. Ruehl, C. Bransley, I. Hartl, Laser Phys. Lett. 13, 1 (2016)

21. H. Fonnum, E. Lippert, M.W. Haakestad, Opt. Lett. 38, 1884 (2013)

22. L.M. Frantz, J.S. Nodvik, J. Appl. Phys. 34, 2346 (1963)

23. W. Koechner: Solid-State Laser Engineering, 4th ed. (SpringerVerlag, 1996), pp. 158-183

24. A.E. Siegman: Lasers (University Science Books, 1986), p. 14

25. AC Materials, 756 Anclote Road, Unit F, Tarpon Springs, Fl 34689 (personal communication, 2014)

26. A. Dergachev, Proc. SPIE 3005, 85990B (2013) 